\title{
The efficacy and roles of combining temozolomide with whole brain radiotherapy in protection neurocognitive function and improvement quality of life of non-small-cell lung cancer patients with brain metastases
}

Xia Deng ${ }^{2+}$, Zhen Zheng ${ }^{2+}$, Baochai Lin², Huafang Su², Hanbin Chen², Shaoran Fei², Zhenghua Fei², Lihao Zhao², Xiance Jin $^{2^{*}}$ and Cong-Ying Xie ${ }^{1,2^{*}}$

\begin{abstract}
Background: Brain metastasis (BM) is a poor prognostic factor for non-small-cell lung cancer (NSCLC). The efficacy and roles of combining temozolomide (TMZ) with whole brain radiotherapy (WBRT) in protection neurocognitive function (NCF) and improvement quality of life (QOL) were investigated and compared with WBRT alone in the treatment of NSCLC patients with BM.

Methods: A total of 238 NSCLC patients with BM were reviewed and categorized into WBRT plus TMZ (RCT) arm and WBRT alone (RT), respectively. The efficacy was evaluated with Pearson chi-square or Fisher's exact tests, Log-rank test and Cox proportional hazards model. NCF was assessed by using revised Hopkins Verbal Learning Test (HVLT-R), Controlled Oral Word Association (COWA) test and Trail-making Test (TMT). QOL was assessed by the Functional Assessment of Cancer Treatment-Lung (FACT-L) Chinese version 4.0 questionnaire.

Results: The average intracranial objective response (ORR) and disease control rate (DCR) for all the patients were 26.9 and $95.8 \%$, respectively. The intracranial ORR and DCR for RCT and RT arm were $34.9 \%$ vs. $20.2 \%(p=0.01)$ and $98.4 \%$ vs. 92.7\% ( $p=0.03$ ), respectively. The median intracranial progression-free survival (PFS) and overall survival (OS) of NSCLC patients with BM were 5.2 and 7.3 months, respectively. The median PFS of RCT arm was significantly longer than that of RT arm (5.9 vs. 4.9 months, $p=0.002$ ). The median OS of the RCT arm was also slightly longer than that of the RT arm (8.5 vs. 5.9 months), but without statistical significance $(p=0.11)$. Multivariate analysis indicated that TMZ was a significant factor for PFS. Statistically significant differences on NCF and QOL were observed between CRT and RT arms at 5 months. RCT showed a trend of toxicities increase compared with RT, however, the toxicities were tolerable and manageable.

(Continued on next page)
\end{abstract}

\footnotetext{
*Correspondence: jinxc1979@hotmail.com; wzxiecongying@163.com

${ }^{\dagger}$ Equal contributors

${ }^{2}$ Department of Radiotherapy and Chemotherapy, the First Affiliated Hospital

of Wenzhou Medical University, No.2 Fuxue Lane, Wenzhou 325000, China

'Zhejiang Provincial Key Laboratory of Aging and Neurological Disorder

Research, Wenzhou 325000, China
} 
(Continued from previous page)

Conclusions: Adding TMZ to WBRT in the treatment of NSCLC patients with BM could improve the intracranial ORR, DCR, and median PFS compared with WBRT alone. Although no remarkable difference on median OS was found, adding TMZ could prevent NCF and QOL from worsening. The side effects increased by adding TMZ, but the difference was not statistical significance and toxicities were well tolerated.

Keywords: Temozolomide, Non-small-cell lung cancer, Brain metastases, Whole brain radiotherapy, Neurocognitive function, Quality of life

\section{Background}

Lung cancer has become the leading cause of cancer related deaths in worldwide [1]. Brain metastasis (BM) is one of the most common complications in non-smallcell lung cancer (NSCLC) patients with more than 10\% patients presented with $\mathrm{BM}$ at their first hospital visit $[2,3]$ and $30-40 \%$ patients developed it during the course of disease [4]. Whole brain radiotherapy (WBRT) is the standard treatment strategy for BM. However, the prognosis of patients with BM remains poor after WBRT with a median overall survival (OS) of 4-6 months. The effect of systemic chemotherapy is limited due to the impenetrability of brain blood barrier [5, 6], as reported that several chemotherapy drugs in combination with WBRT failed to improve the survival [7].

For the past few decades researchers have found that some drugs may have a positive effect on the NSCLC with BM [8-10]. Temozolomide (TMZ) is a new oral alkylating agent, which is able to cross the brain blood barrier with demonstrated survival benefit in the treatment of high-grade gliomas when administered concurrently with adjuvant radiotherapy [11]. Studies demonstrated that TMZ could be used against a broad range of cancers in vitro including NSCLC [12-14]. Adding TMZ to WBRT may improve the response rate of NSCLC patients with BM [15-17]. However, the potential neurocognitive risks and the influence on the patients' living quality of combing TMZ with WBRT were less studied. The purpose of this study is to investigate the survival benefits, neurocognitive function (NCF) and quality of life (QOL) influence of WBRT with or without TMZ in the treatment of NSCLC patients with BM.

\section{Methods}

\section{Patients}

We retrospectively reviewed NSCLC patients with BM treated at the First Affiliated Hospital of Wenzhou Medical University from January 2008 to December 2015. The eligibility criteria for this study were as follows: patients were historically diagnosed with NSCLC and had confirmed BM by magnetic resonance imaging (MRI); had at least one measurable BM with diameter larger than $10 \mathrm{~mm}$; patients had no history of TKI administration; had adequate function of major organs (including cardiac, hepatic, and renal function) and hematologic function (absolute neutrophil $\geq 1.5 \times 10^{9} / \mathrm{L}$ or platelet count $\geq 100 \times 10^{9} / \mathrm{L}$ ); had no uncontrolled morbidities (e.g., myocardial infarction in the last 12 months); with Eastern Cooperative Oncology Group performance status $\leq 3$; Treated by WBRT with a prescription of $3 \mathrm{~Gy} /$ fraction $\times 10$ fractions.

The exclusion criteria were as follows: patients had small cell or mixed small cell histology; patients had EGFR mutations; without at least one measurable lesion according to the Response Evaluation Criteria in Solid Tumors (RECIST) 1.1; lost to follow-up or died within 1 month after starting the treatment; received prior radiotherapy to the brain or TMZ or targeted drugs. This study was carried out according to ethical standards, national and international guidelines. It was approved by the Institutional Review Board and performed at the 1st Affiliated Hospital of Wenzhou Medical University (IRB\#:2015041). Written informed consent was obtained from each patient before treatment.

\section{Treatment schemes}

Patients were divided into WBRT + TMZ (RCT) arm and WBRT (RT) arm, respectively. WBRT was planned with two lateral parallel-opposite conformal beams at a prescription of 30 Gy for 10 fractions with a 6-MV photon beam on an Elekta Synergy linac (Elekta Ltd, Crawley, UK). WBRT plans were delivered through a record and verify system (MosaiQ v. 1.60Q3, IMPAC Medical Systems, Inc., Sunnyvale, CA). In the RCT arm, TMZ $75 \mathrm{mg} / \mathrm{m}^{2} /$ day was administered daily during radiation treatment. After the completion of WBRT, TMZ $100 \mathrm{mg} / \mathrm{m}^{2}$ was continued for 14 days and repeated every 28 days until unacceptable toxicity or disease progression for up to six cycles.

\section{Neurocognitive function and quality of life assessment}

NCF was assessed by using revised Hopkins Verbal Learning Test (HVLT-R), Controlled Oral Word Association (COWA) test and Trail-making Test (TMT). The HVLT-R is a learning and memory test, in which the patient was asked to learn and recall a list of 12 words over three trials [18]. The TMT is a measure of graphomotor speed and set-shifting to measure the executive function [19]. The COWA Test provides a relatively quick test of 
verbal fluency and it is believed to place high demands on executive control processes [20].

QOL was assessed by the Functional Assessment of Cancer Treatment-Lung (FACT-L) Chinese version 4.0 questionnaire, which has 34 items with a 5-point Likert scale [21]. The FACT-L had been shown to be reliable and valid instruments to measure the QOL of Chinese lung cancer patients [22].

\section{Treatment evaluation and follow-up}

The response and progression were evaluated weekly during WBRT. Evaluation included a complete history, neurologic examination, QOL assessment, blood counts, and biochemistry profile. After RT, the evaluation was done monthly for the first 6 month, then every 3 months after. Evaluation included physical examination, neurologic examination, QOL assessment, a complete blood count measurement, liver function test, and chest computed tomography (CT) scan. Brain CT with and without contrast, abdominal CT, or bone scan, as well as MRI if necessary, were performed when there were relevant symptoms in patients.

\section{Definitions and statistical analyses}

Pearson chi-square or Fisher's exact tests (when there were fewer than 5 expected counts in the contingency table) were used to compare the baseline characteristics between RCT and RT arms. Tumor response was assessed according to the Response Evaluation Criteria in Solid Tumors 1.1. OS was defined as the interval from the date of initiation WBRT to the date of death resulted from NSCLC. Intracranial progression-free survival (PFS) was defined as local disease progression, the appearance of new intracranial lesions or both. Intracranial PFS was calculated from the initiation WBRT and the date of confirming progression or death from intracranial progression (if death occurred within 60 days of the last central nervous system assessment date). If the complete survival time of a patient was impossible to obtain or the disease did not progress, patient' status was assumed as the last known survival and/ or contact date. The baseline neurocognitive status was recorded at the first neurocognitive assessment before the start of BM treatment. Adverse events were graded according to the National Cancer Institute Common Terminology Criteria for Adverse Events (NCI-CTCAE) v3.0.

Intracranial PFS and OS were estimated by KaplanMeier method. Differences between groups were compared by the log-rank test. In order to identify risk factors associated with intracranial progression, multivariate analyses were conducted with Cox proportional hazard model. Reliable Change Index was used to categorize the change or improvement on NCF and QOL scores [23]. The Reliable Change Index was derived from the standard error of measurement (SEM) of each test, which is calculated from the test-retest reliability $(\mathrm{r})$ and the standard deviation (SD) of test scores: SEM $=$ SD (1-r) ${ }^{1 / 2}$. The standard error (SE) of difference was then calculated as: $\mathrm{SE}$ diff $=\left[2\left(\mathrm{SEM}^{2}\right)\right]^{12}$. All Reliable Change Index thresholds were rounded to the nearest whole number. Scores in any tests decreased from baseline status and met the Reliable Change Index threshold were categorized as deterioration at a specific time period (e.g. 3 months, 5 months and 7 months). The predictive accuracy of various Cox regression models was quantified by Harrell's concordance index (C-index), which ranges from 0.5 (no predictive power) to 1 (perfect prediction). Statistical analyses were computed using SPSS (version 17.0, SPSS Inc., Chicago, IL) and the R stats package (R Foundation for Statistical Computing, Vienna, Austria). Tests were two sided and $p<0.05$ was considered statistically significant.

\section{Results}

\section{Patients' characteristics}

From January 2008 to December 2015, 485 NSCLC patients with BM were retrospectively reviewed. Seventyeight patients due to loss of follow-up information, 96 patients due to received EGFR Tyrosine Kinase Inhibitor (TKIs) and 39 patients due to without WBRT or did not finish the WBRT were excluded. Nineteen patients had an operation to treat brain metastases and 15 patients died within 1 month after starting WBRT were also excluded (Fig. 1). A total of 238 NSCLC patients with BM were enrolled in this study with a median age of 60 years (range, 34-85). There were 129 patients (54.2\%) and 109 patients (45.8\%) categorized into RCT arm and RT arm, respectively. Baseline characteristics of patients were well balanced between the matched pairs as shown in Table 1.

\section{Responses and survival of patients}

The average intracranial objective response rate (ORR) and disease control rate (DCR) for all patients were $26.9 \%(64 / 238)$ and $95.8 \%$ (228/238), respectively. There were 164 patients $(68.9 \%)$ who had intracranial stable disease and 10 (4.2\%) who had intracranial progressive disease. The intracranial ORR for RCT and RT arm were $34.9 \%(45 / 129)$ vs. $20.2 \%(22 / 109)(p=0.01)$, respectively. The intracranial DCR for RCT was 98.4\% (127/129) compared with $92.7 \%(101 / 129)$ for RT arm $(p=0.03)$.

The median intracranial PFS and OS for all patients were 5.2 months [95\% confidence interval (CI), 4.8-5.6 months] and 7.3 months (95\% Cl, 5.9-8.8 months), respectively. The median intracranial PFS of RCT arm was significantly longer than that of RT arm (5.9 vs. 4.9 months, $p=0.002$ ) (Fig. 2). The estimated 3-month PFS rates were $92.1 \%$ and $87.9 \%$ in the RCT arm and RT arm, respectively. The median OS of the RCT arm was slightly higher than that of the RT arm (8.5 vs. 5.9 months) (Fig. 3), but without statistical significance $(p=0.11)$. The estimated 6- 


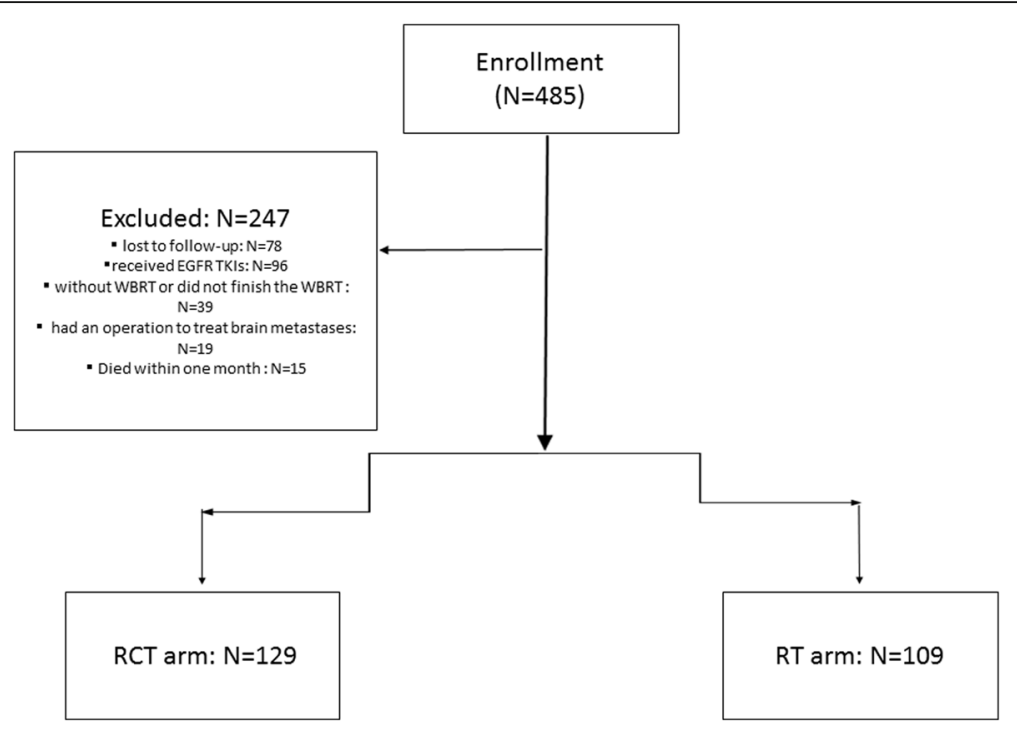

Fig. 1 Flow diagram of patients enrollment

month OS rates were 64.3 and $48.4 \%$ in the RCT and RT arm, respectively.

Table 2 shows the multivariate analysis results on intracranial PFS and OS for all patients with or without the Radiation Therapy Oncology Group (RTOG) recursive partitioning analysis (RPA) classification and graded prognostic assessment (GPA) grade. For multivariate analysis with RPA and GPA included in the Cox's regression model, receiving TMZ $(p=0.004)$, never smoking $(p=0.02)$, primary disease controlled $(p=0.003)$ and lower RTOG RPA class $(p=0.008)$ were of prognostic significance for intracranial PFS. The C-index of this model including smoking status, RTOG RPA class, therapeutic schedule and primary disease situation was 0.726 for intracranial PFS; never smoking $(p=0.03)$, lower RTOG RPA class $(p=0.01)$, RTOG GPA grade $2-4$ $(p=0.02)$ and primary disease controlled $(p<0.001)$ were correlated with longer OS. The $\mathrm{C}$-index of the model was 0.768 for OS. For multivariate analysis without RPA and GPA entering into Cox's regression model, TMZ $(p=0.004)$, smoking status $(p=0.02)$, number of BM $(p=0.02)$ and primary disease controlled $(p=0.007)$ were independent prognostic factors for intracranial PFS. The C-index of this model including smoking status, number of $\mathrm{BM}$, therapeutic schedule and primary disease situation was 0.722 for intracranial PFS; smoking status $(p=0.03)$, number of $\mathrm{BM}(p=0.02)$, performance status (PS) $(p=0.003)$ and primary disease controlled $(p<0.001)$ were associated with OS. The C-index of the model was 0.758 for OS.

\section{Comparison of NCF and QOL}

Table 3 illustrates the compliance to NCF and QOL assessments at the baseline and over the first 7 months of follow-up. There was no significant difference on the compliance between two arms $(p>0.05)$. Table 4 shows the deterioration status over 7 months as defined by Reliable Change Index threshold baseline. Before treatment, there was no significant difference on the declined number of scores for NCF and QOL between two groups $(p>0.05)$. There were 23 out of 105 evaluated patients from RCT arm deteriorated in HVLT-R delayed recall, which were significant lower than $(p=0.02)$ those in RT arm, in which 32 out of 87 were deteriorated. Statistically significant differences were also found in TMTB $(p=0.03)$ and COWA $(p=0.03)$ at 3 months. For HVLT-R and COWA, there were significantly greater deterioration in HVLT-R total recall (TR) $(p=0.008)$, HVLT-R delayed recall $(p=0.007)$, COWA $(p=0.002)$, FACT-L $(p=0.01)$ in the RT arm compared with RCT arm at 5 months. No statistically significant differences between the two arms was observed at 7 months $(p>0.05)$.

\section{Adverse effects}

Side effects comparison between RCT and RT arms were presented in Table 5 . The most frequent hematologic side effects were anemia (55.9\%), neutropenia (52.5\%) and thrombocytopenia (47.1\%). The most common non-hematologic toxicities were nausea $(71.8 \%)$, fatigue $(62.6 \%)$, and vomiting (54.6\%). The common grade III/IV toxicity was nausea (20.6\%). Neutropenia and nausea were the two most frequent grade III/IV hematologic side effects occurred in RCT and RT arms with a rate of $10.1 \%$ vs. $9.2 \%$, and $22.5 \%$ vs. $18.3 \%$, respectively. On the whole, all toxicities were generally brief, reversible, and manageable. They were well tolerated after symptomatic treatments. 
Table 1 Characteristics of NSCLC patients with brain metastasis

\begin{tabular}{|c|c|c|c|c|}
\hline Characteristics & Total (\%) & $\mathrm{RCT}(\%)$ & RT (\%) & $P$ \\
\hline All patients & $238(100)$ & $129(100)$ & $109(100)$ & \\
\hline \multicolumn{5}{|l|}{ Gender } \\
\hline Female & $102(42.9)$ & $60(46.5)$ & $42(38.5)$ & \multirow[t]{2}{*}{0.22} \\
\hline Male & $136(57.1)$ & $69(53.5)$ & $67(61.5)$ & \\
\hline \multicolumn{5}{|l|}{ Smoking } \\
\hline Never & $108(45.4)$ & $63(48.8)$ & $45(41.3)$ & \multirow[t]{2}{*}{0.24} \\
\hline Current/former & $130(54.6)$ & $66(51.2)$ & $64(58.7)$ & \\
\hline \multicolumn{5}{|l|}{ Age } \\
\hline$\leq 60$ & $96(40.3)$ & $58(45.0)$ & 38 (35.9) & \multirow[t]{2}{*}{0.11} \\
\hline$>60$ & $142(59.7)$ & $71(55.0)$ & $71(65.1)$ & \\
\hline \multicolumn{5}{|l|}{ Histology } \\
\hline Adenocarcinoma & $227(95.4)$ & $123(95.3)$ & $104(95.4)$ & \multirow[t]{2}{*}{0.98} \\
\hline Non-adenocarcinoma & $11(4.6)$ & $6(4.7)$ & $5(4.6)$ & \\
\hline \multicolumn{5}{|l|}{ ECOG PS } \\
\hline $0-1$ & $188(79.0)$ & $101(78.3)$ & $87(79.8)$ & \multirow[t]{2}{*}{0.77} \\
\hline $2-3$ & $50(21.0)$ & $28(21.7)$ & $22(20.2)$ & \\
\hline \multicolumn{5}{|l|}{ Prior chemotherapy } \\
\hline NO & $92(38.7)$ & $51(39.5)$ & $41(37.6)$ & \multirow[t]{2}{*}{0.76} \\
\hline YES & $146(61.3)$ & $78(60.5)$ & $68(62.4)$ & \\
\hline \multicolumn{5}{|l|}{ Number of BM } \\
\hline$\leq 3$ & $65(27.3 \%)$ & $33(25.6 \%)$ & $32(29.4 \%)$ & \multirow[t]{2}{*}{0.52} \\
\hline$>3$ & $173(72.7 \%)$ & $96(74.4 \%)$ & 77 (70.6\%) & \\
\hline \multicolumn{5}{|l|}{ Extracranial metastases } \\
\hline NO & $93(39.1 \%)$ & $48(37.2 \%)$ & $45(41.3 \%)$ & \multirow[t]{2}{*}{0.52} \\
\hline YES & 145 (60.9\%) & 81 (62.8\%) & $64(58.7 \%)$ & \\
\hline \multicolumn{5}{|l|}{ Primary disease control } \\
\hline NO & 31 (13.0\%) & $20(15.5 \%)$ & $11(10.1 \%)$ & \multirow[t]{2}{*}{0.22} \\
\hline YES & 207 (87.0\%) & 109 (84.5\%) & 98 (89.9\%) & \\
\hline \multicolumn{5}{|l|}{ RTOG RPA class } \\
\hline Classl & 50 (21.0\%) & 31 (24.0\%) & 19 (17.4\%) & \multirow[t]{2}{*}{0.21} \\
\hline Class II + III & 188 (79.0\%) & 98 (76.0\%) & $90(82.6 \%)$ & \\
\hline \multicolumn{5}{|l|}{ RTOG GPA grade } \\
\hline $0-2$ & 143 (60.1\%) & 74 (57.4\%) & $69(63.3 \%)$ & \multirow[t]{2}{*}{0.35} \\
\hline $2.5-4$ & 95 (39.9\%) & 55 (42.6\%) & $40(36.7 \%)$ & \\
\hline
\end{tabular}

Abbreviations: Eastern Cooperative Oncology Group performance status ECOG $P S$, Brain metastasis $B M$, the Radiation Therapy Oncology Group RTOG, recursive partitioning analysis $R P A$, graded prognostic assessment GPA

\section{Discussion}

The effects and influence on Neurocognitive function and QOL of adding TMZ to WBRT in the treatment of NSCLC with BM were investigated in a total of 238 patients. Our study suggested that TMZ combined with WBRT could significantly enhance the intracranial ORR and DCR, as well as median PFS compared with WBRT alone in the treatment of NSCLC patients with BM, but no remarkable difference on median OS was found.

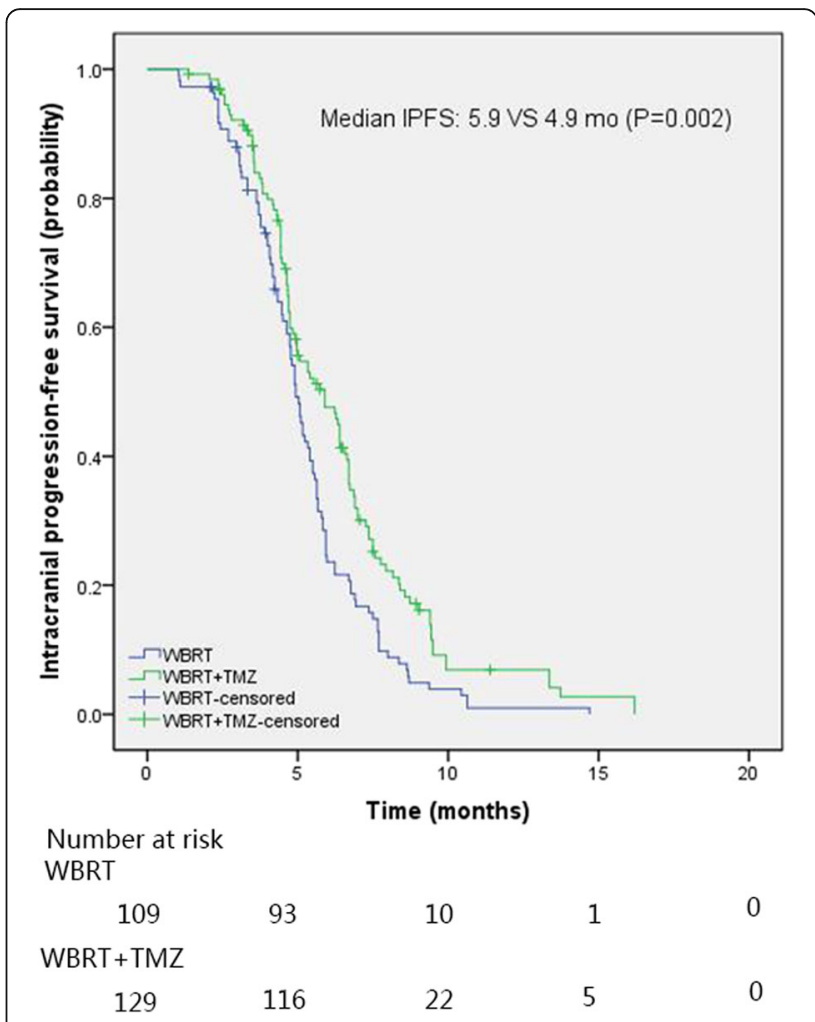

Fig. 2 The intracranial progression-free survival of NSCLC patients with brain metastases

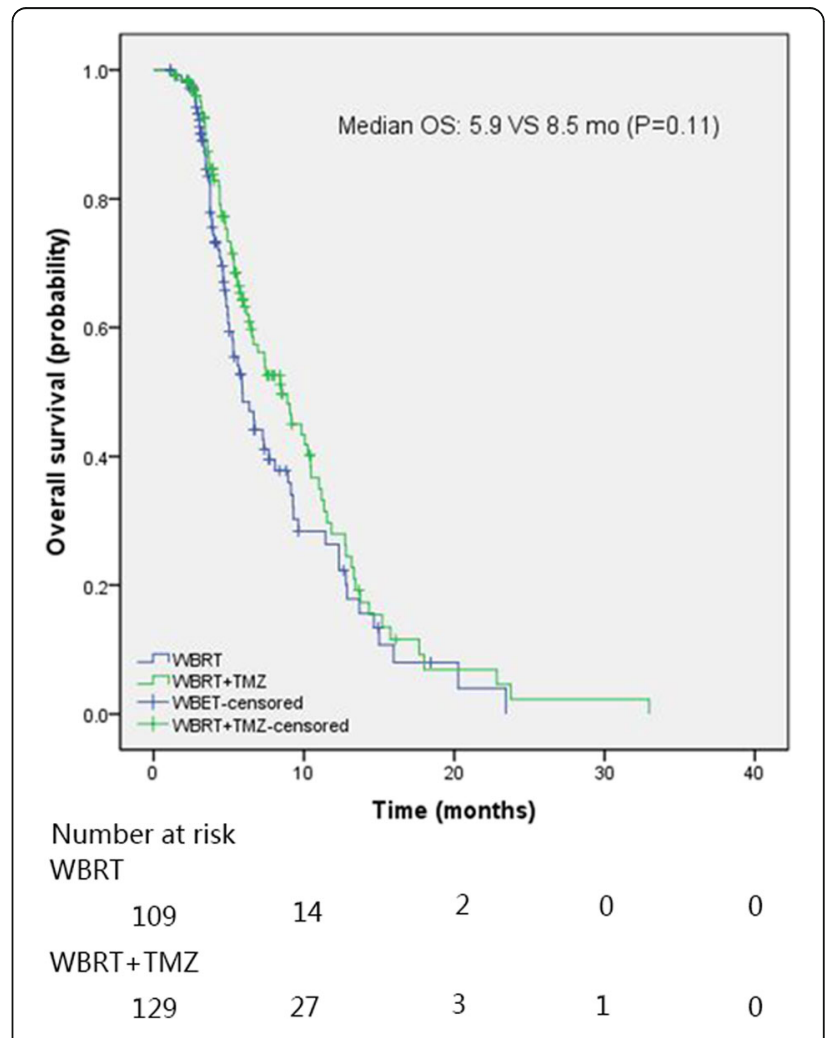

Fig. 3 The overall survival of NSCLC patients with brain metastases 
Table 2 Multivariate analysis of factors affecting intracranial PFS and OS in NSCLC patients with brain metastasis

\begin{tabular}{|c|c|c|c|c|c|c|c|}
\hline \multirow[t]{2}{*}{ Factors } & \multicolumn{4}{|c|}{ Intracranial PFS } & \multicolumn{3}{|l|}{ OS } \\
\hline & $\mathrm{N}$ & $\mathrm{HR}$ & $95 \% \mathrm{Cl}$ & $P$ & $\mathrm{HR}$ & $95 \% \mathrm{Cl}$ & $P$ \\
\hline \multicolumn{8}{|l|}{ With RPA and GPA in the model } \\
\hline Smoking & $108 / 130$ & 1.42 & $1.07-1.88$ & 0.02 & 1.48 & $1.04-2.10$ & 0.03 \\
\hline Primary disease controlled & $31 / 207$ & 0.54 & $0.36-0.81$ & 0.003 & 0.36 & $0.23-0.57$ & $<0.001$ \\
\hline RTOG RPA class & $50 / 188$ & 1.81 & $1.17-2.79$ & 0.008 & 1.99 & $1.15-3.45$ & 0.01 \\
\hline Therapeutic schedule & $109 / 129$ & 0.66 & $0.50-0.88$ & 0.004 & & & \\
\hline RTOG GPA grade & $143 / 95$ & & & & 0.58 & $0.36-0.93$ & 0.02 \\
\hline \multicolumn{8}{|c|}{ Without RPA and GPA in the model } \\
\hline Smoking & $108 / 130$ & 1.39 & $1.04-1.84$ & 0.02 & 1.48 & $1.05-2.10$ & 0.03 \\
\hline Number of BM & $65 / 173$ & 1.47 & $1.08-2.00$ & 0.02 & 1.58 & $1.08-2.33$ & 0.02 \\
\hline Primary disease controlled & $31 / 207$ & 0.57 & $0.38-0.86$ & 0.007 & 0.38 & $0.24-0.60$ & $<0.001$ \\
\hline Therapeutic schedule & $109 / 129$ & 0.66 & $0.50-0.88$ & 0.004 & & & \\
\hline EGOC PS & $188 / 50$ & & & & 1.76 & $1.21-2.57$ & 0.003 \\
\hline
\end{tabular}

Abbreviations: RPA recursive partitioning analysis, GPA graded prognostic assessment, $B M$ brain metastasis, Eastern Cooperative Oncology Group performance status ECOG PS, the Radiation Therapy Oncology Group RTOG, confidence interval $\mathrm{Cl}$, hazard ratio $H R$

NCF and QOL were also prevented from worsening by adding TMZ.

In this study, the intracranial ORR and DCR of NSCLC patients with BM treated by WBRT + TMZ were 34.9 and $98.4 \%$, respectively, which were significantly higher than 20.2 and $92.7 \%$ in the RT arm (both $p<0.05$ ). These were consistent with results reported in previous studies that TMZ + WBRT may enhance the overall ORR of NSCLC patients with BM compared with WBRT alone $[23,24]$. A multi-institutional trial showed a higher overall

Table 3 Neurocognitive and quality of life assessment compliance

\begin{tabular}{|c|c|c|c|c|c|}
\hline \multirow{2}{*}{$\begin{array}{l}\text { Evaluation } \\
\text { Status }\end{array}$} & \multicolumn{2}{|l|}{ RCT arm } & \multicolumn{2}{|l|}{ RT arm } & \multirow[t]{2}{*}{$P$} \\
\hline & Not evaluated & Received & Not evaluated & Received & \\
\hline \multicolumn{6}{|c|}{ Hopkins Verbal Learning Test (HVLT-R) } \\
\hline Baseline & 5 & 124 & 4 & 105 & 0.93 \\
\hline At 3 Months & 24 & 105 & 32 & 87 & 0.12 \\
\hline At 5 Months & 57 & 72 & 55 & 64 & 0.75 \\
\hline At 7 Months & 87 & 42 & 83 & 36 & 0.70 \\
\hline \multicolumn{6}{|c|}{ Trail-making Test (TMT) } \\
\hline Baseline & 6 & 123 & 4 & 105 & 0.71 \\
\hline At 3 Months & 26 & 103 & 31 & 88 & 0.27 \\
\hline At 5 Months & 59 & 70 & 57 & 62 & 0.73 \\
\hline At 7 Months & 85 & 44 & 83 & 36 & 0.52 \\
\hline \multicolumn{6}{|c|}{ Controlled Oral Word Association (COWA) test } \\
\hline Baseline & 5 & 124 & 6 & 103 & 0.55 \\
\hline At 3 Months & 24 & 105 & 30 & 89 & 0.21 \\
\hline At 5 Months & 57 & 72 & 58 & 61 & 0.47 \\
\hline At 7 Months & 85 & 44 & 85 & 34 & 0.35 \\
\hline \multicolumn{6}{|c|}{ Functional Assessment of Cancer Treatment-Lung (FACT-L) } \\
\hline Baseline & 7 & 122 & 9 & 100 & 0.48 \\
\hline At 3 Months & 27 & 102 & 34 & 85 & 0.16 \\
\hline At 5 Months & 56 & 73 & 54 & 65 & 0.76 \\
\hline At 7 Months & 88 & 41 & 86 & 33 & 0.49 \\
\hline
\end{tabular}

Abbreviations: Hopkins Verbal Learning Test HVLT-R, Trail-making Test TMT, Controlled Oral Word Association test COWA, Functional Assessment of Cancer Treatment-Lung FACT-L 
Table 4 Deterioration status from baseline in each examination using reliable change index

\begin{tabular}{|c|c|c|c|c|c|}
\hline \multirow{2}{*}{$\begin{array}{l}\text { Deterioration } \\
\text { status }\end{array}$} & \multicolumn{2}{|l|}{ RCT arm } & \multicolumn{2}{|l|}{ RT arm } & \multirow[t]{2}{*}{$p$} \\
\hline & Deterioration & No deterioration & Deterioration & No deterioration & \\
\hline \multicolumn{6}{|l|}{ At 3 months } \\
\hline HVLT-R TR & 21 & 84 & 19 & 68 & 0.76 \\
\hline HVLT-R DR & 23 & 82 & 32 & 55 & 0.02 \\
\hline TMT Part A & 22 & 81 & 25 & 63 & 0.26 \\
\hline TMT Part B & 24 & 79 & 33 & 55 & 0.03 \\
\hline COWA & 19 & 86 & 28 & 61 & 0.03 \\
\hline FACT-L & 23 & 79 & 24 & 61 & 0.45 \\
\hline \multicolumn{6}{|l|}{ At 5 months } \\
\hline HVLT-R TR & 21 & 511 & 33 & 31 & 0.008 \\
\hline HVLT-R DR & 23 & 49 & 35 & 29 & 0.007 \\
\hline TMT Part A & 18 & 52 & 25 & 37 & 0.07 \\
\hline TMT Part B & 21 & 49 & 30 & 32 & 0.03 \\
\hline COWA & 19 & 53 & 32 & 29 & 0.002 \\
\hline FACT-L & 25 & 48 & 36 & 29 & 0.01 \\
\hline \multicolumn{6}{|l|}{ At 7 months } \\
\hline HVLT-R TR & 20 & 22 & 19 & 17 & 0.65 \\
\hline HVLT-R DR & 24 & 18 & 17 & 19 & 0.38 \\
\hline TMT Part A & 23 & 21 & 21 & 15 & 0.59 \\
\hline TMT Part B & 25 & 19 & 22 & 14 & 0.70 \\
\hline COWA & 24 & 20 & 20 & 14 & 0.71 \\
\hline FACT-L & 24 & 17 & 21 & 12 & 0.66 \\
\hline
\end{tabular}

Abbreviations: HVLT-R TR Hopkins Verbal Learning Test total recall, HVLT-R DR Hopkins Verbal Learning Test delayed recall, TMT Trail-making Test, COWA Controlled Oral Word Association, FACT-L Functional Assessment of Cancer Treatment-Lung

ORR (48\% vs. $27 \%, p=0.03$ ) in 103 lung cancer patients with BM treated with TMZ $75 \mathrm{mg} / \mathrm{m}^{2}$ per day plus WBRT compared with WBRT alone [24]. Through a meta-analysis, Liao Kai et al. also reported that WBRT + TMZ could significantly improve ORR (risk ratio = $1.55, p=0.003)$ in the treatment of BM from NSCLC compared with WBRT alone [23]. However, a phase II trial reported that adding TMZ to WBRT did not improve the ORR compared with WBRT alone for 12 chemotherapy-native NSCLC patients with BM [25]. In another phase II trial, for 30 pre-treated recurrent NSCLC patients with BM treated by concurrent WBRT and TMZ $\left(150-200 \mathrm{mg} / \mathrm{m}^{2} / \mathrm{d}\right)$, only $3(10)$ and $6(20 \%)$ patients achieved an objective response and disease control [26]. We inferred that pretreatment influenced the efficacy of TMZ in these phase II patients.

The median OS for all NSCLC patients with BM observed in this study was 7.3 months, which is close to

Table $\mathbf{5}$ Toxicity profile for the NSCLC with brain metastasis patients treated by CRT and RT

\begin{tabular}{|c|c|c|c|c|c|c|}
\hline \multirow[t]{2}{*}{ Side effects/N (\%) } & \multicolumn{2}{|c|}{ RCT $\operatorname{arm}(N=129)$} & \multicolumn{2}{|c|}{ RT $\operatorname{arm}(N=109)$} & \multirow{2}{*}{$\begin{array}{l}P \text { for all } \\
\text { grades }\end{array}$} & \multirow{2}{*}{$\begin{array}{l}P \text { for grade } \\
\text { III/IV }\end{array}$} \\
\hline & All grades & Grade III/IV & All grades & Grade III/IV & & \\
\hline Fatigue & $81(62.8)$ & $16(12.4)$ & $68(62.4)$ & $12(11.0)$ & 0.95 & 0.74 \\
\hline Anorexia & $64(49.6)$ & $14(10.9)$ & $47(43.1)$ & $9(8.3)$ & 0.29 & 0.50 \\
\hline Diarrhea & $18(13.9)$ & $0(0 \%)$ & $12(11.0)$ & $0(0 \%)$ & 0.50 & NA \\
\hline Nausea & $88(68.2)$ & $29(22.5)$ & $83(76.1)$ & $20(18.3)$ & 0.18 & 0.43 \\
\hline Vomiting & $69(53.5)$ & $14(10.9)$ & $61(56.0)$ & $13(11.9)$ & 0.70 & 0.80 \\
\hline Headache & 55 (42.6) & $13(10.1)$ & $43(39.4)$ & $11(10.1)$ & 0.62 & 0.99 \\
\hline Anemia & $72(55.8)$ & $5(3.9)$ & $61(56.0)$ & $3(2.8)$ & 0.98 & 0.91 \\
\hline Neutropenia & $66(51.2)$ & $13(10.1)$ & $59(54.1)$ & $10(9.2)$ & 0.65 & 0.81 \\
\hline Thrombocytopenia & $61(47.3)$ & $4(3.1)$ & $51(46.8)$ & $2(1.8)$ & 0.94 & 0.84 \\
\hline
\end{tabular}


the reported median OS of 8.0 months in the study of Wang Q et al., in which NSCLC patients with BM were treated by WBRT followed by intensity-modulated boost combined with concomitant TMZ [16]. In this study, the median OS and PFS in the WBRT + TMZ group and in the WBRT alone group were 8.5 vs. 5.9 months and 5.9 vs. 4.9 months, respectively. Daniel Chua et al. also demonstrated that WBRT + TMZ had a higher median OS (5.7 vs. 4.4 months) and PFS (3.8 vs. 3.1 months) compared with WBRT alone in the treatment of NSCLC patients with BM [27]. However, their reported median OS and PFS were inferior than ours. We speculated that the difference may resulted from different TMZ doses were administered in two studies. In the study of Daniel Chua, patients received TMZ daily for 21 days, while in our study, TMZ $75 \mathrm{mg} / \mathrm{m}^{2} /$ day was administered daily during radiation treatment and TMZ $100 \mathrm{mg} / \mathrm{m}^{2} /$ day was continued for 14 days and repeated every 28 days until unacceptable toxicity or disease progression for up to six cycles.

Previous studies reported that TMZ combing with RT could improve QOL in high grade glioma [28, 29]. A single-institution phase I clinical trial on patients with multiple brain lesions from breast carcinoma treated by capecitabine and TMZ demonstrated that significant improvements in attention span $(p=0.047)$ and emotional function $(p=0.016)$ were observed indicating that adding TMZ was not neurotoxic and may have a beneficial effect [30]. Addeo R et al. also reported that a statistically significant improvement on QOL was found at 3,6 and 9 months for 59 patients treated by 30 Gy WBRT with concomitant TMZ [31]. Similarly, our result implied that adding TMZ in the treatment of NSCLC patients with BM could prevent the NCF and QOL from worsening at 5 months. These studies implied that TMZ as a maintenance therapy may improve patients' NCF and QOL. This may due to a better intracranial ORR and DCR in RCT group. TMZ may has a certain function of preventing tumor recurrence in brain.

Nausea and fatigue were the most frequent side effects observed for both RCT and RT arms, followed by anemia, vomiting, neutropenia, anorexia and thrombocytopenia, etc. Addition TMZ in the RCT arm showed a trend of increasing the rate of side effects compared with RT alone, as reported in previous studies [27, 32]. However, the difference of the adverse events occurrence between RCT and RT arms was not statistically significant.

One limitation of current study is that it is a retrospective methodology from a single institution experience. The impact of various treatments related outcomes could not be fully evaluated. The number of patients enrolled may not be sufficient enough and the follow-up duration of the study may not be long enough. External validation using other large database for further evaluating the prognostic effect of adding TMZ in the treatment of NSCLC patients with $\mathrm{BM}$ would be of great value in clinical practice.

\section{Conclusion}

In a conclusion, adding TMZ to WBRT in the treatment of NSCLC patients with BM could improve the intracranial ORR and DCR, as well as median PFS compared with WBRT alone. However, no remarkable difference on median OS was found. NCF and QOL were also prevented from worsening by adding TMZ. Although the side effects were increased by adding TMZ, the difference was not statistical significance and they were well tolerated.

\section{Abbreviations}

BM: Brain metastasis; Cl: Confidence interval; COWA: Controlled oral word association; CT: Computed tomography; DCR: Disease control rate; FACT$L:$ the Functional assessment of cancer treatment-lung; GPA: Graded prognostic assessment; HVLT-R: Revised Hopkins verbal learning test: MRI: Magnetic resonance imaging; NCF: Neurocognitive function; NSCLC: Non-small-cell lung cancer; ORR: Objective response rate; OS: Overall survival; PFS: Progression-free survival; PS: Performance status; QOL: Quality of life; RPA: Recursive partitioning analysis; RTOG: the Radiation therapy oncology group; TKIs: Tyrosine kinase inhibitor; TMT: Trail-making test; TMZ: Temozolomide; WBRT: Whole brain radiotherapy

\section{Acknowledgements \\ None.}

\section{Funding}

This study was partly supported by funding in data collection and analysis from Zhejing Provincial Key Laboratory of Aging and Neurological Disorder Research (LH013); Zhejiang Provincial Natural Foundation (LY16H160047), National Natural Foundation (11675122) and Health Science and Technology Funding of Zhejiang Provincial Health Department (2015KYB241).

\section{Availability of data and materials}

All the data was included in the manuscript.

\section{Authors' contributions}

XD and ZZ carried out data analyses and drafted the manuscript. BL, HS, LZ did the followup; HC, FS, ZF performed the statistical analysis. XJ, CX designed, coordinated, and supervised the study and critically reviewed and discussed the manuscript. All authors have read and approved the final version of the manuscript.

\section{Competing interests}

The authors declare that they have no competing interests.

Consent for publication

Not applicable.

\section{Ethics approval and consent to participate}

This study was carried out according to ethical standards, national and international guidelines. It was approved by the Institutional Review Board and performed at the 1st Affiliated Hospital of Wenzhou Medical University (IRB\#:2015041). Written informed consent was obtained from each patient before treatment.

Received: 18 May 2016 Accepted: 16 December 2016

Published online: 10 January 2017

\section{References}

1. Steuer CE, Ramalingam SS. Targeting EGFR in lung cancer: Lessons learned and future perspectives. Mol Asp Med. 2015;45:67-73.

2. Langer CJ, Mehta MP. Current management of brain metastases, with a focus on systemic options. J Clin Oncol Off J Am Soc Clin Oncol. 2005; 23(25):6207-19. 
3. Eichler AF, Loeffler JS. Multidisciplinary management of brain metastases. Oncologist. 2007;12(7):884-98.

4. Huang Q, Ouyang X. Predictive biochemical-markers for the development of brain metastases from lung cancer: clinical evidence and future directions. Cancer Epidemiol. 2013;37(5):703-7.

5. Postmus PE, Smit EF. Chemotherapy for brain metastases of lung cancer: a review. Ann Oncol. 1999;10(7):753-9.

6. Moscetti L, Nelli F, Felici A, Rinaldi M, De Santis S, D'Auria G, Mansueto G, Tonini G, Sperduti I, Pollera FC. Up-front chemotherapy and radiation treatment in newly diagnosed nonsmall cell lung cancer with brain metastases: survey by Outcome Research Network for Evaluation of Treatment Results in Oncology. Cancer. 2007;109(2):274-81.

7. Mehta MP, Paleologos NA, Mikkelsen T, Robinson PD, Ammirati M, Andrews DW, Asher AL, Burri SH, Cobbs CS, Gaspar LE, et al. The role of chemotherapy in the management of newly diagnosed brain metastases: a systematic review and evidence-based clinical practice guideline. J Neuro-Oncol. 2010;96(1):71-83.

8. Liu R, Wang X, Ma B, Yang K, Zhang Q, Tian J. Concomitant or adjuvant temozolomide with whole-brain irradiation for brain metastases: a metaanalysis. Anti-Cancer Drugs. 2010;21(1):120-8.

9. Sperduto PW, Wang M, Robins HI, Schell MC, Werner-Wasik M, Komaki R, Souhami L, Buyyounouski MK, Khuntia D, Demas W, et al. A phase 3 trial of whole brain radiation therapy and stereotactic radiosurgery alone versus WBRT and SRS with temozolomide or erlotinib for non-small cell lung cancer and 1 to 3 brain metastases: Radiation Therapy Oncology Group 0320. Int J Radiat Oncol Biol Phys. 2013;85(5):1312-8.

10. Welsh JW, Komaki R, Amini A, Munsell MF, Unger W, Allen PK, Chang JY, Wefel JS, McGovern SL, Garland LL, et al. Phase II trial of erlotinib plus concurrent whole-brain radiation therapy for patients with brain metastases from non-smallcell lung cancer. J Clin Oncol Off J Am Soc Clin Oncol. 2013;31(7):895-902.

11. Stupp R, Mason WP, van den Bent MJ, Weller M, Fisher B, Taphoorn MJ, Belanger K, Brandes AA, Marosi C, Bogdahn U, et al. Radiotherapy plus concomitant and adjuvant temozolomide for glioblastoma. N Engl J Med. 2005;352(10):987-96.

12. Raymond E, Izbicka E, Soda H, Gerson SL, Dugan M, Von Hoff DD. Activity of temozolomide against human tumor colony-forming units. Clin Cancer Res. 1997;3(10):1769-74.

13. Middlemas DS, Stewart CF, Kirstein MN, Poquette C, Friedman HS, Houghton PJ, Brent TP. Biochemical correlates of temozolomide sensitivity in pediatric solid tumor xenograft models. Clin Cancer Res. 2000;6(3):998-1007.

14. Srivenugopal KS, Shou J, Mullapudi SR, Lang Jr FF, Rao JS, Ali-Osman F. Enforced expression of wild-type p53 curtails the transcription of the $O$ (6)-methylguanineDNA methyltransferase gene in human tumor cells and enhances their sensitivity to alkylating agents. Clin Cancer Res. 2001;7(5):1398-409.

15. Robins HI, O'Neill A, Mehta M, Grossman S. A phase 3 trial of whole brain radiation therapy and stereotactic radiosurgery alone versus WBRT \& SRS with temozolomide or erlotinib for non-small cell lung cancer and 1 to 3 brain metastases: Radiation Therapy Oncology Group 0320: in regard to Sperduto et al. Int J Radiat Oncol Biol Phys. 2013;86(5):809-10.

16. Wang Q, Jiang Z, Qi X, Lu S, Wang S, Leng C, Lu F, Liu H, Liang S, Shi J. Whole brain radiation therapy followed by intensity-modulated boosting treatment combined with concomitant temozolomide for brain metastases from non-small-cell lung cancer. Clin Transl Oncol. 2014;16(11):1000-5.

17. Minniti G, Scaringi C, Lanzetta G, Bozzao A, Romano A, De Sanctis V, Valeriani M, Osti M, Enrici RM. Whole brain reirradiation and concurrent temozolomide in patients with brain metastases. J Neuro-Oncol. 2014;118(2):329-34.

18. Benedict R, Schretlen D, Groninger L, Brandt J. Hopkins Verbal Learning Test-Revised: Normative Data and Analysis of Inter-Form and Test-Retest Reliability. Clin Neuropsychol. 1998;12(1):43-55.

19. Hirota C, Watanabe M, Sun W, Tanimoto Y, Kono R, Takasaki K, Kono K. Association between the Trail Making Test and physical performance in elderly Japanese. Geriatr Gerontol Int. 2010;10(1):40-7.

20. Ruff RM, Light RH, Parker SB, Levin HS. Benton Controlled Oral Word Association Test: reliability and updated norms. Arch Clin Neuropsychol. 1996;11(4):329-38.

21. Cella D. The Functional Assessment of Cancer Therapy-Lung and Lung Cancer Subscale assess quality of life and meaningful symptom improvement in lung cancer. Semin Oncol. 2004;31(3 Suppl 9):11-5.

22. Wan C, Zhang C, Cai L, Tu X, Feng C, Luo J, Zhang X. Psychometric properties of the Chinese version of the FACT-L for measuring quality of life in patients with lung cancer. Lung Cancer. 2007;56(3):415-21.

23. Liao K, Bi ZF, He Y, Liu YM. Whole brain radiation therapy plus temozolomide in the treatment of brain metastases from non small cell lung cancer: a meta-analysis. Zhonghua yi xue za zhi. 2012;92(45):3199-203.
24. Antonadou D, Coliarakis N, Paraskevaidis M, Athanasiou H, Sarris G, Synodinou M, Skarlatos L, Georgakopoulos G, Beroukas C, Karageorgis P, et al. O-67 A multi-institutional trial comparing survival of patients with brain metastases from lung cancer treated with temozolomide plus radiotherapy versus to radiotherapy alone. Lung Cancer. 2003;41 Suppl 2:S22-3.

25. Dziadziuszko R, Ardizzoni A, Postmus PE, Smit EF, Price A, Debruyne C, Legrand C, Giaccone G, Group ELC. Temozolomide in patients with advanced non-small cell lung cancer with and without brain metastases. a phase II study of the EORTC Lung Cancer Group (08965). Eur J Cancer. 2003;39(9):1271-6.

26. Lauren P. The Two Histological Main Types of Gastric Carcinoma: Diffuse And So-Called Intestinal-Type Carcinoma. An Attempt at a Histo-Clinical Classification. Acta Pathol Microbiol Scand. 1965;64:31-49.

27. Chua D, Krzakowski M, Chouaid C, Pallotta MG, Martinez Jl, Gottfried M, Curran W, Throuvalas N. Whole-brain radiation therapy plus concomitant temozolomide for the treatment of brain metastases from non-small-cell lung cancer: a randomized, open-label phase II study. Clin Lung Cancer. 2010;11(3):176-81.

28. Minniti G, Scaringi C, Baldoni A, Lanzetta G, De Sanctis V, Esposito V, Enrici RM. Health-related quality of life in elderly patients with newly diagnosed glioblastoma treated with short-course radiation therapy plus concomitant and adjuvant temozolomide. Int J Radiat Oncol Biol Phys. 2013;86(2):285-91.

29. Choong NW, Mauer AM, Hoffman PC, Rudin CM, Winegarden 3rd JD, Villano $J$, Kozloff M, Wade 3rd JL, Sciortino DF, Szeto L, et al. Phase II trial of temozolomide and irinotecan as second-line treatment for advanced non-small cell lung cancer. J Thorac Oncol. 2006;1(3):245-51.

30. Rivera E, Meyers C, Groves M, Valero V, Francis D, Arun B, Broglio K, Yin G, Hortobagyi GN, Buchholz T. Phase I study of capecitabine in combination with temozolomide in the treatment of patients with brain metastases from breast carcinoma. Cancer. 2006;107(6):1348-54.

31. Addeo R, Caraglia M, Faiola V, Capasso E, Vincenzi B, Montella L, Guarrasi R, Caserta L, Del Prete S. Concomitant treatment of brain metastasis with whole brain radiotherapy [WBRT] and temozolomide [TMZ] is active and improves quality of life. BMC Cancer. 2007;7:18.

32. Hassler MR, Pfeifer W, Knocke-Abulesz TH, Geissler K, Altorjai G, Dieckmann $\mathrm{K}$, Marosi C. Temozolomide added to whole brain radiotherapy in patients with multiple brain metastases of non-small-cell lung cancer: a multicentric Austrian phase II study. Wien Klin Wochenschr. 2013;125(15-16):481-6.

\section{Submit your next manuscript to BioMed Central and we will help you at every step:}

- We accept pre-submission inquiries

- Our selector tool helps you to find the most relevant journal

- We provide round the clock customer support

- Convenient online submission

- Thorough peer review

- Inclusion in PubMed and all major indexing services

- Maximum visibility for your research

Submit your manuscript at www.biomedcentral.com/submit
Biomed Central 